\title{
Sleep-immune system interaction: advantages and challenges of human sleep loss model
}

\section{Md Dilshad Manzar and M. Ejaz Hussain*}

Sleep Research Group, Centre for Physiotherapy and Rehabilitation Sciences, Jamia Millia Islamia, New Delhi, India *Correspondence: ejaz58@yahoo.com

\section{INTRODUCTION}

Sleep and its functional interaction with immune system is well recognized (Majde and Krueger, 2005; Krueger and Majde, 2011). Sleep synchronized changes in brain activity are implicated in direct rather than indirect immune function (Bryant et al., 2004). Sleep or at least its homeostatic component has been suggested to have an active auto-regulatory and/or auto-modulatory mechanism (Krueger et al., 2008; Kumar, 2010). This mechanism operates through adenosine and other sleep regulating substances (SRSs) like interleukin-1 beta (IL-1 $\beta$ ), tumor necrosis factor-alpha (TNF- $\alpha$ ), growth-hormone-releasing hormone (GHRH), corticotropin-releasing hormone (CRH), nitric oxide (NO), prostaglandin $\mathrm{D}_{2}$ (PGD2), etc. These SRSs are most likely the connecting link between sleep and immune system (Krueger et al., 2008). The issue of peripheral cytokines affecting brain signaling in sleep has been reviewed comprehensively with three suggested routes for transfer of peripheral cytokines to the brain (Krueger and Majde, 2003). These routes may also be involved in transfer and hence in transmitting the signals from peripheral SRSs. Sleep-immune system co-relations has been investigated across a wide range of immune parameters out of which cytokines, immune cells, antibodies, and neuro-endocrine system constitute the focal group. Human sleep loss (SL) models have been of great help in mechanistic characterization of cytokines (IL-1 $\beta$ and TNF- $\alpha$ role) in sleep and neuroendocrine components (GHRH and CRH) in non-REM sleep, respectively (Majde and Krueger, 2005; Krueger and Majde, 2011). The paradigm has helped establish sleep's functional characterization because of practical simplicity (Reynolds and Banks, 2010) and often non-ambiguous results. However, there may be certain limitations with SL model in context of result translation pertaining to different parameter patterns. The comment has been surmised to highlight these challenges which may give direction to future research in minimizing the variable factors. It may also help to compare the results from available literature objectively.

\section{SLEEP LOSS MODEL: ADVANTAGES AND CHALLENGES}

Sleep loss is one of the models which have been employed to study sleep-immune system inter-relationship. It is subdivided into three categories namely total sleep deprivation (TSD), chronic sleep restriction/ partial sleep deprivation (PSD), and sleep fragmentation/disruption. TSD (both acute and chronic variations) and PSD have been used widely, however, sleep disruption has been very rarely employed (Reynolds and Banks, 2010). Human SL model has helped in establishing sleep-immune interaction and is practically hassle free in planning. The outcomes of these studies have usually been non-ambiguous. Various groups have reported similar trends with disparities, mainly because of differences in SL design (Majde and Krueger, 2005; Krueger and Majde, 2011). For instance, sTNFR p55 level did not alter on PSD (Haack et al., 2007; Boudjeltia et al., 2008) but it increased on three nights of TSD (Haack et al., 2007). Sometimes, PSD and acute TST (one/two night) studies have yielded similar trends like in case of white blood cell (WBC; Dinges et al., 1994; Boudjeltia et al., 2008; Liu et al., 2009), neutrophil (Boudjeltia et al., 2008; Liu et al., 2009), and serum IL-1ß (Frey et al., 2007; van Leeuwen et al., 2009).

Human sleep deprivation studies have been asserted not to have the problem of stress-induced forced wakefulness as is done in animal experiments (Dinges et al., 1994; Redwine et al., 2004; Krueger and Majde, 2011). There was no behavio$\mathrm{ral} /$ physiological symptom of stress after one night of TSD, though there was some evidence of neurobehavioral stress after $63 \mathrm{~h}$ without sleep (Dinges et al., 1994). The study has quoted seven articles on
glucocorticoid-SL interaction pattern and concluded that SL without physical/ mental demand is not affected by stress in laboratory conditions. The study further illustrated an increase in peripheral total WBC, granulocytes, monocytes, and natural killer cells activity with striking consistency, irrespective of gender, race, or time of the year (Dinges et al., 1994). However, a more recent article cited non-uniformity (TSD effect) on cortisol pattern, with three studies reporting an increase, three reporting a decrease, and seven reporting no change. It also reported a significant increase in subjective stress with $40 \mathrm{~h}$ TSD (Frey et al., 2007). Moreover, the co-relationship between stress and blood pressure (Gasperin et al., 2009) and increase in blood pressure on TSD with different SL designs has been reported by three studies (Meier-Ewert et al., 2004). Similarly, increase in heart rate, suggesting stress during PSD, has also been reported (van Leeuwen et al., 2009). The inconclusiveness of the evidences vis-a-vis cortisol pattern and SL; subjective stress and SL; and stress-blood pressure-SL leave little to doubt about elusiveness of consensus on the issue of stress-SL interaction. Most importantly, there is very significant gap in the literature in terms of SL study data of children, adolescent, and elderly. It is may be more than interesting for future studies to delve into the dimension, which may open new dimension in context of SL effect on stress marker(s).

Many other elements have also been implicated by other groups to affect the immune variables during SL studies. The duration of SL, circadian phase, posture control, light exposure, blood sampling frequency, nutrition (type and timing), assay sensitivity, body mass index (BMI), and obesity may limit comparability of SL results. These factors were enumerated to explain the disparities in reports of interleukin-6 (IL-6), C-reactive protein (CRP), and cortisol outcome by different groups (Frey 
et al., 2007). The blood samplings through intravenous catheter limit the investigation of diurnal variations in immune functions. The intravenous catheter induces local alterations in cytokines and soluble cytokine receptors production (Haack et al., 2000). IL-6 level increased with the use of an intravenous catheter across $24 \mathrm{~h}$ regardless of sleep deprivation (Haack et al., 2002). The volunteer age and gender had also been shown and/or indicated to affect sleep deprivation effect on some immune variables. The activation of cellular inflammation markers (IL-6 and TNF- $\alpha$ ) on PSD is gender dependent (Irwin et al., 2010). The contrasting pattern of monocyte in young men and postmenopausal women may be attributed to age and gender (Boudjeltia et al., 2008). The likely influence of sleep deprivation on melatonin amplitude has been described with the possible involvement of age (Zeitzer et al., 2007). The finding is very important because melatonin and its' rhythm dynamics has intricate co-relationship with cortisol, catecholamine, and other rhythm markers (Manzar and Hussain, 2011). This may have entailments for circadian pattern of immune parameter and their variations with SL designs. PSD decreased Mac-1 positive lymphocytes; and increased L-selectin positive lymphocytes and monocytes. However, the differences were discernible at 06:30 h and not at 03:00 hours. This may reflect more of sleep rebound (homeostatic-sleep expression) than PSD effect (Redwine et al., 2004).

The auto-regulatory/auto-modulatory characteristic of sleep and/or its homeostatic part (Krueger et al., 2008; Kumar, 2010) operate through adenosine and other SRSs like IL- $1 \beta$, TNF- $\alpha$, GHRH, CRH, NO, PGD2, etc. The majority of these mediators are either cytokine or endocrine components. The colligating attribute of SRSs in the sleep-immune interplay has been described (Krueger et al., 2008). The indication of three routes for transfer and hence transmittance of peripheral (production site) SRSs borne signal to brain foregrounds the sleep-immune system relation (Krueger and Majde, 2003). Sleep regulation depends on the interplay of three elements namely circadian, homeostatic, and allostatic components (Saper et al., 2005). Their involvement in SL study results have been connoted and/or evidenced (Redwine et al., 2004; McEwen, 2006; Zeitzer et al., 2007). Hence, it is advisable to keep these generalizations in consideration during comparison of SL study results and/or designing future studies:

(i) Interaction between specific SL design with circadian, homeostatic, and allostatic characteristic of sleep.

(ii) Implications for the rhythm dynamics of immune parameter being investigated with the design of SL study.

(iii) The differences in design of TSD, PSD, and sleep disruption studies from different laboratories.

Furthermore, there are some studies which suggest inhibition of hippocampal neurogenesis, independent of adrenal stress hormones on prolonged SL, with the possibility of cognitive dysfunction and mood disorders. Even, modest sleep restriction may interfere with neurogenesis enhancement associated with learning processes (Meerlo et al., 2009). Future studies should address the ethical issues of exposing the volunteers to chronic SL and appropriate remedial management like cognitive function assessment and mood analysis tests.

The opinion highlighted the advantages like practical simplicity; often nonambiguous results, lack of consensus on the issue of stress non-interference, important gap in literature on stress-SL interaction, challenges complicating generality, and future directions. All factors implicated to affect the result should be taken into consideration while comparing results from other and/or older studies. The opinion may give directions to future studies to minimize and/ or clearly report about outcomes affecting factors in literature; filling in of literature gap; and some ethical management.

\section{ACKNOWLEDGMENTS}

The work was funded by Indian Council of Medical Research in the form of SRF to Md Dilshad Manzar.

\section{REFERENCES}

Boudjeltia, K. Z., Faraut, B., Stenuit, P., Esposito, M. J., Dyzma, M., Brohée, D., Ducobu, J., Vanhaeverbeek, M., and Kerkhofs, M. (2008). Sleep restriction increases white blood cells, mainly neutrophil count, in young healthy men: a pilot study. Vasc. Health Risk Manag. 4, 1467-1470.

Bryant, P. A., Trinder, J., and Curtis, N. (2004). Sick and tired: does sleep have a vital role in the immune system? Nat. Rev. Immunol. 4, 457-467.

Dinges, D. F., Douglas, S. D., Zaugg, L., Campbell, D. E., McMann, J. M., Whitehouse, W. G., Orne, E.
C., Kapoor, S. C., Icaza, E., and Orne, M. T. (1994). Leukocytosis and natural killer cell function parallel neurobehavioral fatigue induced by 64 hours of sleep deprivation. J. Clin. Invest. 93, 1930-1939.

Frey, D. J., Fleshner, M., and Wright, K. P. Jr. (2007). The effects of 40 hours of total sleep deprivation on inflammatory markers in healthy young adults. Brain Behav. Immun. 21, 1050-1057.

Gasperin, D., Netuveli, G., Dias-da-Costa, J. S., and Pattussi, M. P. (2009). Effect of psychological stress on blood pressure increase: a metaanalysis of cohort studies. Cad. Saude Publica 25, 715-726.

Haack, M., Kraus, T., Schuld, A., Dalal, M., Koethe, D., and Pollmächer, T. (2002). Diurnal variations of interleukin-6 plasma levels are confounded by blood drawing procedures. Psychoneuroendocrinology 27, 921-931.

Haack, M., Reichenberg, A., Kraus, T., Schuld, A., Yirmiya, R., and Pollmächer, T. (2000). Effects of an intravenous catheter on the local production of cytokines and soluble cytokine receptors in healthy men. Cytokine 12, 694-698.

Haack, M., Sanchez, E., and Mullington, J. M. (2007). Elevated inflammatory markers in response to prolonged sleep restriction are associated with increased pain experience in healthy volunteers. Sleep 30, 1145-1152.

Irwin, M. R., Carrillo, C., and Olmstead, R. (2010). Sleep loss activates cellular markers of inflammation: sex differences. Brain Behav. Immun. 24, 54-57.

Krueger, J. M., and Majde, J. A. (2003). Humoral links between sleep and the immune system: research issues. Ann. N. Y. Acad. Sci. 992, 9-20.

Krueger, J. M., and Majde, J. A. (2011). "Sleep and host defense," in Principles and Practice of Sleep Medicine, eds M. H. Kryger, T. Roth, and W. C. Dement (St. Louis, MO: Saunders), 261-290.

Krueger, J. M., Rector, D. M., Roy, S., Van Dongen, H. P., Belenky, G., and Panksepp, J. (2008). Sleep as a fundamental property of neuronal assemblies. Nat. Rev. Neurosci. 9, 910-919.

Kumar, V. M. (2010). Sleep is neither a passive nor an active phenomenon. Sleep Biol. Rhythms 8, 163-169.

Liu, H., Wang, G., Luan, G., and Liu, Q. (2009). Effects of sleep and sleep deprivation on blood cell count and hemostasis parameters in healthy humans. J. Thromb. Thrombolysis 28, 46-49.

Majde, J. A., and Krueger, J. M. (2005). Links between the innate immune system and sleep. J. Allergy Clin. Immunol. 116, 1188-1198.

Manzar, D., and Hussain, M.E. (2011). Leptin rhythmicity and its relationship with other rhythm markers. Biol. Rhythm Res. 42, 163-180.

McEwen, B. S. (2006). Sleep deprivation as a neurobiologic and physiologic stressor: allostasis and allostatic load. Metab. Clin. Exp. 55, S20-S23.

Meerlo, P., Mistlberger, R. E., Jacobs, B. L., Heller, H. C., and McGinty, D. (2009). New neurons in the adult brain: the role of sleep and consequences of sleep loss. Sleep Med. Rev. 13, 187-194.

Meier-Ewert, H. K., Ridker, P. M., Rifai, N., Regan, M. M., Price, N. J., Dinges, D. F., and Mullington, J. M. (2004). Effect of sleep loss on C-reactive protein, an inflammatory marker of cardiovascular risk. J. Am. Coll. Cardiol. 43, 678-683.

Redwine, L., Dang, J., and Irwin, M. (2004). Cellular adhesion molecule expression, nocturnal sleep, and 
partial night sleep deprivation. Brain Behav. Immun. $18,333-340$

Reynolds, A. C., and Banks, S. (2010). Total sleep deprivation, chronic sleep restriction and sleep disruption. Prog. Brain Res. 185, 91-103.

Saper, C. B., Scammell, T. E., and Lu, J. (2005). Hypothalamic regulation of sleep and circadian rhythms. Nature 437. 1257-1263.

van Leeuwen, W. M., Lehto, M., Karisola, P., Lindholm, H., Luukkonen, R., Sallinen, M., Härmä, M., Porkka-Heiskanen, T., and Alenius, H. (2009). Sleep restriction increases the risk of developing cardiovascular diseases by augmenting proinflammatory responses through IL-17 and CRP. PLoS ONE4, e4589. doi: 10.1371/journal.pone.0004589

Zeitzer, J. M., Duffy, J. F., Lockley, S. W., Dijk, D. J., and Czeisler, C. A. (2007). Plasma melatonin rhythms in young and older humans during sleep, sleep deprivation, and wake. Sleep 30, 1437-1443.

Received: 15 September 2011; accepted: 03 January 2012; published online: 16 January 2012.
Citation: Manzar MD and Hussain ME (2012) Sleepimmune system interaction: advantages and challenges of human sleep loss model. Front. Neur. 3:2. doi: 10.3389/ fneur.2012.00002

This article was submitted to Frontiers in Sleep and Chronobiology, a specialty of Frontiers in Neurology.

Copyright $\odot 2012$ Manzar and Hussain. This is an open-access article distributed under the terms of the Creative Commons Attribution Non Commercial License, which permits non-commercial use, distribution, and reproduction in other forums, provided the original authors and source are credited. 